See Article page 48.

\section{Commentary: The mitral valve and the left ventricle in functional mitral regurgitation: The puppet and the puppeteer}

\author{
Dongyang $\mathrm{Xu}, \mathrm{MS},{ }^{\mathrm{a}}$ and Muralidhar Padala, $\mathrm{PhD}^{\mathrm{a}, \mathrm{b}}$
}

The mitral valve is a beautiful structure, whose function depends upon an intricate force balance between the valve and the underlying left ventricle. When the ventricle falters, such as in cardiomyopathies, the mitral valve is perturbed and it regurgitates; and when the valve fails, such as in mitral valve prolapse, the ventricle is perturbed from the volume overload and it fails. Functional or ischemic mitral regurgitation (FMR/IMR) is mitral valve failure due to ventricular disease, wherein dilatation of the left ventricle secondary to myocardial ischemia or infarction perturbs the mitral valve. The mitral valve leaflets are tethered in systole, leading to loss of coaptation and FMR/IMR. Although widely cited, ${ }^{1,2}$ this mechanism of ventricular dilatation causing valve tethering, does not explain the heterogeneity in the prevalence of FMR in ischemic or postinfarction cardiomyopathy patients ( $\sim 40 \%$ have FMR) who uniformly have perturbed and dilated left ventricles. Also the heterogenous prevalence of FMR in heart transplant candidates with severely dilated and dysfunctional ventricles does not conform to the prevailing hypothesis. Two schools of alternative mechanisms have since evolved-(1) the hypothesis that mitral valve leaflets grow in response to tethering from the dilated ventricle, and FMR/IMR exists in patients in whom such leaflet growth is stunted ${ }^{3}$; and (2) the hypothesis that FMR/IMR occurs when the dynamic interpapillary muscle shortening is

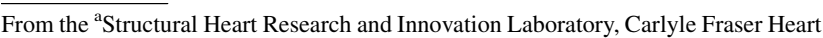
Center, Emory University Hospital Midtown; and ${ }^{\mathrm{b}}$ Division of Cardiothoracic Surgery, Emory University School of Medicine, Atlanta, Ga.

Disclosures: The authors reported no conflicts of interest.

The Journal policy requires editors and reviewers to disclose conflicts of interest and to decline handling or reviewing manuscripts for which they may have a conflict of interest. The editors and reviewers of this article have no conflicts of interest.

Received for publication Nov 24, 2020; revisions received Nov 24, 2020; accepted for publication Nov 27, 2020; available ahead of print Dec 28, 2020.

Address for reprints: Muralidhar Padala, PhD, 380B Northyards Blvd, Atlanta, GA 30313 (E-mail: spadala@emory.edu).

JTCVS Open 2021;5:63-5

2666-2736

Copyright (C) 2020 The Authors. Published by Elsevier Inc. on behalf of The American Association for Thoracic Surgery. This is an open access article under the CC BY-NCND license (http://creativecommons.org/licenses/by-nc-nd/4.0/).

https://doi.org/10.1016/j.xjon.2020.11.011
}

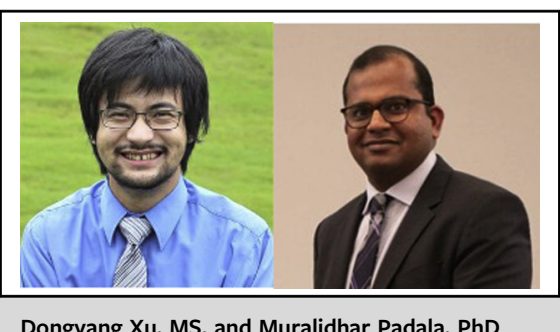

Dongyang $\mathrm{Xu}, \mathrm{MS}$, and Muralidhar Padala, PhD

CENTRAL MESSAGE

Mechanisms of ischemic mitral

regurgitation are explained using multimodality imaging in a sheep

model.

perturbed, independent of the left ventricular size. ${ }^{4}$ The first hypothesis has spawned research into the molecular mechanisms defining leaflet growth in response to tethering, and the potential to use targeted therapeutics to modulate this mechanism. Despite unabated enthusiasm for this hypothesis, ${ }^{5-9}$ there are no data that confirm that leaflets grow (ie, increase in size, mass, and cellularity) and not plainly elongate, as most soft tissues do, when tethered. The second hypothesis has spawned research into restoring interpapillary muscle dimensions with papillary muscle approximation as a simple surgical or interventional approach to restore physiological force balance and correct the regurgitation. ${ }^{10-14}$ Since elevated systolic interpapillary muscle separation and reduced diastolic-to-systolic interpapillary muscle shortening can stretch the mitral leaflet edges and restrict the formation of a vertical coaptation shelf, a technique to approximate the muscles may ameliorate valve dysfunction.

Aly and colleagues, ${ }^{15}$ in this issue of the Journal, characterize both mechanisms in an ovine model of postinfarction cardiomyopathy. This laboratory has been at the forefront in using sheep to define the mechanistic basis for FMR/IMR for several decades, ${ }^{16,17}$ and this new contribution towards characterizing the aforementioned alternative mechanisms causing FMR/IMR in this ovine model are indeed valuable. Six sheep underwent ligation of the left circumflex artery distal to the first obtuse marginal branch, and imaging was used to quantify valvular and ventricular changes until 12 weeks after the infarction. The salient findings of the study may be summarized as follows:

- the mitral annulus dilated over the 12 weeks after infarction, with an increase in annular area $(20 \%)$, annular circumference $(11 \%)$, septal-lateral annular diameter 
$(11 \%)$, and commissure-to-commissure diameter $(9.3 \%)$. The annular perturbations are relatively small, and thus it may be reasonable to attribute the FMR/ IMR to leaflet or ventricular mechanisms over annular perturbations in this model. However, in humans, the extent of annular dilatation may be more severe.

- the saddle shape of the mitral annulus was preserved despite the dilatation, which is counter to previous literature from this group and others, ${ }^{18,19}$ which emphasized the need to restore the saddle-shaped mitral annulus in FMR/IMR. These data may indicate that annular ring shape may not matter in the setting of this disease, if annuloplasty is used. However, annular flattening may occur in humans.

- tenting volume and index were significantly elevated by 12 weeks. When the contribution of each leaflet cusp to the tenting volume is examined, measured as tethering angle, both anterior and posterior leaflets were restricted. However, the magnitude of restriction of the anterior leaflet was lower $(\mathrm{A} 1-86 \%$ reduction, $\mathrm{A} 2-66 \%$ reduction, A3-84\% reduction) compared with the posterior leaflet $(\mathrm{P} 1-110 \%$ reduction, $\mathrm{P} 2-162 \%$ reduction, and $\mathrm{P} 3-506 \%$ reduction). This is expected, as ligation of the left circumflex artery distal to the first obtuse marginal would induce a posterolateral myocardial infarction that would include the posteromedial papillary muscle and the entire underlying wall to become infarcted. Thus, restriction of leaflet mobility is more severe in the leaflet cusps supported by chordae from the infarcted papillary muscle (A3-P3). Surprisingly, this excessive tethering is only seen in the posterior P3 cusp, not the A3 cusp. It is possible that despite equal tethering forces on both leaflet cusps, larger area of the anterior A3 cusp enables higher, counteracting closing forces for a given transmitral pressure, than the smaller P3 cusp is unable to generate. Thus, the resulting tethering angle in the anterior A3 cusp may be lower than the posterior P3 cusp. An increase in tethering angle at cusps remote to the infarcted papillary muscle can be attributed to the global increase in end-diastolic volume over the 12-week period. In our experience, tethering angles do not capture tenting adequately, as the leaflets are often curved differently from the annulus to the coaptation.

- the total leaflet area was significantly increased at 12 weeks compared with the baseline. However, such an increase in the leaflet area was dominated by anterior leaflet area increase and surprisingly a reduction in the posterior leaflet area. It is unclear from the manuscript if the leaflet areas and lengths were measured in the unloaded diastolic state, but assuming such is the case, this is an interesting finding. The anterior and posterior leaflets differ significantly in their composition and material properties ${ }^{20,21}$ and thus may have different responses to tethering. The anterior leaflet with its more organized matrix, may have better extensibility than the posterior leaflet, which may lack such extensibility. Therefore, for a given pathological stretch magnitude, it is possible that the anterior leaflet expands, but the posterior leaflet lacks extensibility and thus develops higher stresses that cause fibrosis and shrinkage. Although speculative, there is some evidence in recent literature that imposing unphysiological strains on the mitral valve can cause fibrosis, via activation of mechanosensitive pathways. ${ }^{10,22}$

- the new framework in which papillary muscle displacement was investigated in relation to the mitral annular plane, and in relation to each other is laudable. When examining the changes in the relative distances between the papillary muscles and the mitral annular land marks, there are some salient findings. The noninfarcted anterolateral papillary muscle was displaced only in relation to the posterior commissure but not any other landmarks, at end diastole. This is likely from the dilatation of the annulus around the posterior commissure and not from displacement of this papillary muscle away from the mitral annular plane. At end systole, this observation was preserved, in addition to a significant rise in the distance between the anterolateral papillary muscle and the mid anterior annulus. On the contrary, the infarcted posteromedial papillary muscle was displaced in both end diastole and end systole from the posterior commissure, mid-point of the anterior annulus, mid-point of the posterior annulus, center of the mitral annulus, and the anterior commissure. Part of the displacement may be from atrophy of the papillary muscle itself, part from outward displacement of the lateral infarcted wall, and part from lack of contraction and radial inward displacement of the muscle in systole. These observations are expected, and corroborate previous findings from the same group using sonomicrometry. These data, if extrapolated to surgical repair techniques used to correct FMR, indicate that the Kron procedure ${ }^{23}$ (hoisting the posteromedial papillary muscle towards the posterior commissure) may have some merit. Whether such hoisting is beneficial, when performed to the mid-point of the anterior annulus, or the midpoint of the posterior annulus, or the anterior commissure is unclear and unpredictable, warranting further investigation. The authors suggestion that hoisting the muscle toward the mid-point of the anterior or posterior annulus has merit.

- The interpapillary muscle separation was distinctly increased, both at end diastole and at end systole, at 12 weeks after the infarction. At end diastole, the separation between the muscles increased by $23.5 \%$ from baseline to 12 weeks after infarction. At end systole, the 
separation between the muscles increased by $61.65 \%$ from baseline to 12 weeks after infarction. Clearly, the lateral separation between the muscles is increased not only because of ventricular dilatation but also because of poor radial contraction in the equatorial segments of the left ventricle, where the papillary muscles insert. The authors identify that the lateral separation between the muscles is initially elevated due to reduced motion between the 2 papillary muscles (bilateral congruent loss of motion), which emerges into a unilateral phenomenon wherein the infarcted papillary muscle becomes stationary in relation to the other papillary muscle. Using cardiac magnetic resonance imaging in patients with and without FMR, our group demonstrated that loss of such interpapillary muscle dynamics is a strong predictor of FMR than ventricular size. ${ }^{4}$ Evidence from this animal model validates the clinical hypothesis and provides a well-characterized animal model to develop surgical techniques that can target this perturbation. Recently, papillary muscle approximation has gained some traction, as it abolishes this lateral separation between the muscles. Hvass and colleagues ${ }^{24}$ first reported this technique in a manner that the muscles were approximated at their bases with an encircling graft, which was recently used in a randomized study by Nappi and colleagues. ${ }^{12}$ The results were significantly better with such an approach, but in both cases an undersizing ring was also used. In another study, the papillary muscle tips or entire lengths were approximated, which yielded a better result. ${ }^{13}$ However, an important consideration in implementing this technique is that each of the papillary muscles must be drawn individually into the mitral orifice and approximated, rather than drawing one papillary muscle toward another stationary one. The former approach enables balance of forces on both sides of the mitral valve, which the later approach cannot and may cause tethering on one half and prolapse on the other.

In summary, the authors must be commended for characterizing the geometric perturbations of the mitral valve and the left ventricle in this sheep model, which can be used to develop new repair strategies. We thus congratulate them for a nicely done study.

\section{References}

1. Levine RA, Hagege AA, Judge DP, Padala M, Dal-Bianco JP, Aikawa E, et al. Mitral valve disease-morphology and mechanisms. Nat Rev Cardiol. 2015; 12:689-710.

2. He S, Fontaine AA, Schwammenthal E, Yoganathan AP, Levine RA. Integrated mechanism for functional mitral regurgitation: leaflet restriction versus coapting force: in vitro studies. Circulation. 1997;96:1826-34.

3. Dal-Bianco JP, Aikawa E, Bischoff J, Guerrero JL, Handschumacher MD, Sullivan S, et al. Active adaptation of the tethered mitral valve: insights into a compensatory mechanism for functional mitral regurgitation. Circulation. 2009; 120:334-42.

4. Kalra K, Wang Q, McIver BV, Shi W, Guyton RA, Sun W, et al. Temporal changes in interpapillary muscle dynamics as an active indicator of mitral valve and left ventricular interaction in ischemic mitral regurgitation. J Am Coll Cardiol. 2014;64:1867-79.

5. Beaudoin J, Dal-Bianco JP, Aikawa E, Bischoff J, Guerrero JL, Sullivan S, et al. Mitral leaflet changes following myocardial infarction: clinical evidence for maladaptive valvular remodeling. Circ Cardiovasc Imaging. 2017;10:e006512.

6. Nakai H, Kaku K, Takeuchi M, Otani K, Yoshitani H, Haruki N, et al. Different influences of left ventricular remodeling on anterior and posterior mitral leaflet tethering. Circ J. 2012;76:2481-7.

7. Messas E, Bel A, Szymanski C, Cohen I, Touchot B, Handschumacher MD, et al. Relief of mitral leaflet tethering following chronic myocardial infarction by chordal cutting diminishes left ventricular remodeling. Circ Cardiovasc Imaging. 2010;3:679-86.

8. Chaput M, Handschumacher MD, Guerrero JL, Holmvang G, Dal-Bianco JP, Sullivan S, et al. Mitral leaflet adaptation to ventricular remodeling: prospective changes in a model of ischemic mitral regurgitation. Circulation. 2009;120: S99-103.

9. Chaput M, Handschumacher MD, Tournoux F, Hua L, Guerrero JL, Vlahakes GJ, et al. Mitral leaflet adaptation to ventricular remodeling: occurrence and adequacy in patients with functional mitral regurgitation. Circulation. 2008;118: 845-52.

10. Sielicka A, Sarin EL, Shi W, Sulejmani F, Corporan D, Kalra K, et al. Pathological remodeling of mitral valve leaflets from unphysiologic leaflet mechanics after undersized mitral annuloplasty to repair ischemic mitral regurgitation. J Am Heart Assoc. 2018;7:e009777.

11. Padala M. Papillary muscle approximation is an anatomically correct repair for ischemic mitral regurgitation. J Am Coll Cardiol. 2016;68:1146-7.

12. Nappi F, Lusini M, Spadaccio C, Nenna A, Covino E, Acar C, et al. Papillary muscle approximation versus restrictive annuloplasty alone for severe ischemic mitral regurgitation. J Am Coll Cardiol. 2016;67:2334-46.

13. Wakasa S, Kubota S, Shingu Y, Ooka T, Tachibana T, Matsui Y. The extent of papillary muscle approximation affects mortality and durability of mitral valve repair for ischemic mitral regurgitation. J Cardiothorac Surg. 2014;9:98.

14. Rama A, Praschker L, Barreda E, Gandjbakhch I. Papillary muscle approximation for functional ischemic mitral regurgitation. Ann Thorac Surg. 2007;84:2130-1.

15. Aly AH, Saito Y, Bouma W, Pilla JJ, Pouch AM, Yushkevich PA, et al. Multimodal image analysis and subvalvular dynamics in ischemic mitral regurgitation. J Thorac Cardiovasc Surg Open. 2021;5:48-60.

16. Gorman JH III, Gorman RC, Jackson BM, Hiramatsu Y, Gikakis N, Kelley ST, et al. Distortions of the mitral valve in acute ischemic mitral regurgitation. Ann Thorac Surg. 1997;64:1026-31.

17. Gorman RC, McCaughan JS, Ratcliffe MB, Gupta KB, Streicher JT, Ferrari VA, et al. Pathogenesis of acute ischemic mitral regurgitation in three dimensions. $J$ Thorac Cardiovasc Surg. 1995;109:684-93.

18. Mahmood F, Gorman JH III, Subramaniam B, Gorman RC, Panzica PJ, Hagberg RC, et al. Changes in mitral valve annular geometry after repair: saddle-shaped versus flat annuloplasty rings. Ann Thorac Surg. 2010;90: $1212-20$.

19. Gorman JH III, Jackson BM, Enomoto Y, Gorman RC. The effect of regional ischemia on mitral valve annular saddle shape. Ann Thorac Surg. 2004;77:544-8.

20. Ranganathan N, Lam JH, Wigle ED, Silver MD. Morphology of the human mitral valve. II. The value leaflets. Circulation. 1970;41:459-67.

21. May-Newman K, Yin FC. Biaxial mechanical behavior of excised porcine mitral valve leaflets. Am J Physiol. 1995;269:H1319-27.

22. Howsmon DP, Rego BV, Castillero E, Ayoub S, Khalighi AH, Gorman RC, et al Mitral valve leaflet response to ischaemic mitral regurgitation: from gene expression to tissue remodelling. J R Soc Interf. 2020;17:20200098.

23. Kron IL, Green GR, Cope JT. Surgical relocation of the posterior papillary muscle in chronic ischemic mitral regurgitation. Ann Thorac Surg. 2002;74: 600-1.

24. Hvass U, Tapia M, Baron F, Pouzet B, Shafy A. Papillary muscle sling: a new functional approach to mitral repair in patients with ischemic left ventricular dysfunction and functional mitral regurgitation. Ann Thorac Surg. 2003;75: 809-11. 